\title{
Malakoplakia associated with adrenal and islet cell hyperplasia and glucagonoma
}

\author{
John McClure, D.S. Fairweather, ${ }^{1}$ A.R. Mene and S.S. Banerjee \\ Departments of Pathology, and 'Geriatric Medicine, University of Manchester, Manchester, UK.
}

\begin{abstract}
Summary: Extensive involvement of the urinary tract and related structures by malakoplakia presented difficult diagnostic problems in two elderly individuals. At post-mortem examination there was bilateral adrenal cortical hyperplasia in both patients and, in one, there was also a pancreatic glucagonoma and diffuse hyperplasia of the pancreatic islet tissues. There is now an increasing body of evidence that malakoplakia is associated with hypercorticosteroidism possibly due to an effect on macrophage exocytotic mechanisms. The coexistence of malakoplakia and glucagonoma has not been previously described.
\end{abstract}

\section{Introduction}

Malakoplakia is an uncommon granulomatous condition characterized by the aggregation of large, granular, eosinophilic cells (von Hansemann cells) which contain typical intracytoplasmic inclusions called Michaelis-Gutmann (MG) bodies. ${ }^{1}$ These are between 5 and $10 \mu \mathrm{m}$ in diameter and exhibit concentric laminations so that they are variously described as being 'targetoid' or 'owl's eye' in appearance. MG bodies always contain calcium and phosphate in the form of hydroxyapatitite crystals and, not infrequently, quantities of iron. ${ }^{2}$ The most common site of malakoplakia is the mucosa of the urinary tract especially its lower part ${ }^{3,4,5}$ and there is a strong association with urinary tract infections by coliform organisms. $^{6}$

Before 1958 malakoplakia had only been described within the confines of the urinary tract and renal parenchyma. Since then the condition has been described not only in structures relatively close to these, including testis, ${ }^{7}$ prostate ${ }^{8}$ and retroperitoneum ${ }^{9}$ but also in more remote sites such as brain, ${ }^{10}$ lung, ${ }^{11}$ bone $^{12}$ and gastrointestinal tract. ${ }^{13}$ These cases of malakoplakia outside the urinary tract often show extensive involvement of more than one organ and frequently exhibit a systemic disorder in which the background is often one of immunosuppression or hypercortico-steroidism. ${ }^{6,14,15}$

Although malakoplakia of the urinary tract is predominantly a disease of women in their fifth decade

Correspondence: J. McClure, B.Sc., M.D., M.R.C.Path., Department of Histopathology, UHSM, Nell Lane, West Didsbury, Manchester M20 8LR, UK.

Accepted: 23 December 1986. it also has a high incidence in older age groups. It occurs much less frequently in males. ${ }^{6}$ Clinically malakoplakia may present complex, challenging diagnostic and therapeutic problems. This may be due to the absolute rarity of the condition, relative lack of awareness of it and its association with other disease processes. Recently we have had experience of malakoplakia in two elderly patients in whom the diagnosis was not clarified until post-mortem examination and in whom there was morphological evidence of endocrine abnormalities.

\section{Case reports}

\section{Patient 1}

A 73 year old man was admitted with an ill-defined history of weight loss over 8 weeks and intermittent diarrhoea. He was thin $(59 \mathrm{~kg})$ and there was nonpalpable purpura on both legs which rapidly progressed to form haemorrhagic sheets. Skin biopsy showed sub-epithelial bullae and a minimal inflammatory infiltrate with no evidence of vasculitis. The bleeding time was prolonged to 9.5 minutes, but coagulation tests were normal as was the white cell ascorbate level. The purpura resolved over several weeks.

Urinalysis persistently showed significant bacteriuria, pyuria and desquamated epithelial cells. Despite several courses of appropriate antibiotics, his urine remained infected with $E$. coli, proteus and enterococci species, and he had two episodes of septicaemia. He later developed an abscess in the left groin which was drained surgically. Despite intensive 
treatment with antibiotics and intravenous fluids, he gradually deteriorated and died 5 months after his initial presentation.

At post-mortem examination the body weighed $54 \mathrm{~kg}$ (182 cm in length). A sinus was present in the left groin communicating with an ill-defined mass arising in the prostate and extending onto the left pelvic wall. The urinary bladder contained purulent urine. Both kidneys were scarred and finely granular. There was colonic diverticular disease and the rectum was adherent to the prostatic lesion. The adrenal glands were enlarged with a combined weight of $70 \mathrm{~g}$.

Microscopically the inguinal sinus, and the related prostatic mass, was a malakoplakic lesion containing numerous large histiocytes (von Hansemann cells) with intracytoplasmic inclusions (Michaelis-Gutmann bodies). These were of a typical targetoid appearance, contained calcium salts (Figure 1) and iron and exhibited PAS positivity (diastase resistance). There was bilateral adrenal cortical nodular hyperplasia. The pituitary was microscopically normal. Death was due to generalized microscopic pulmonary embolism from thrombosis of the periprostatic venous plexus.

\section{Patient 2}

An 81 year old female was admitted with malaise and confusion. Her physical deterioration had begun one year previously. She had no relevant past medical history. Examination and investigation showed severe megaloblastic anaemia (haemoglobin $4.0 \mathrm{~g} / \mathrm{dl}$ ) due to vitamin $B_{12}$ deficiency.

Four days after admission she developed haematuria and acute urinary retention. An intravenous urogram showed bilateral hydronephrosis. At cystoscopy the bladder epithelium was almost entirely covered by a white membrane. Coliforms, Proteus

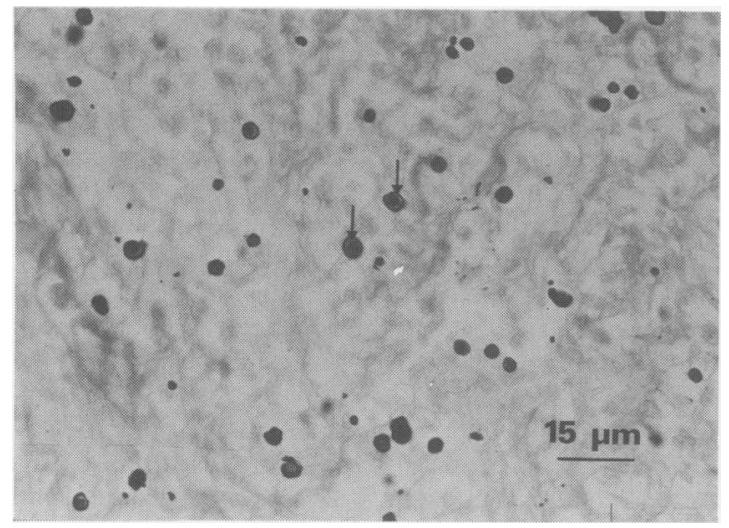

Figure 1 Typical Michaelis-Gutmann (MG) bodies are arrowed (von Kossa/light green). species and enterococci were frequently cultured from the urine. Appropriate antibiotics were administered but with no lasting effects. She had intermittent pyrexia and leucocytosis. Her catheter became blocked because of further bleeding and she required blood transfusion. Two months after admission she developed acute renal failure and died.

At post-mortem examination the body weighed $41 \mathrm{~kg}(156 \mathrm{~cm}$ in length). There was an extensive bilateral bronchopneumonia. Both kidneys were swollen but the capsules stripped easily. There was severe gangrenous pyelitis with marked destruction of the calyces. The tips of the renal papillae were necrotic. The pelves and ureters were lined by a thick fibrinopurulent membrane. The urinary bladder was dilated and there was a transmural necrosis with the formation of a pseudomembrane on the mucosal aspect of the bladder. Loops of small intestine were adherent to the outer surface of the bladder. Both adrenals were enlarged, the combined weight being $80 \mathrm{~g}$. The cut surfaces showed nodularity and were slightly haemorrhagic.

Microscopically there was a severe mixed inflammatory process involving the bladder mucosa containing large numbers of von Hansemann cells with MG bodies. These showed typical staining reactions and contained calcium and iron salts. Malakoplakic infilo trates were present in the mucosa of the ureters and the urinary pelves. There was bilateral renal papillarye necrosis and acute pyelonephritis. There was no. evidence of renal malakoplakia. There was bilateral nodular adrenal cortical hyperplasia. In the pancreas there was prominence of islet tissue and a single islet cell tumour (Figure 2). This was $1 \mathrm{~cm}$ in diameter and composed of trabeculae of uniform cells related to an appreciable vasculature. Immunocytochemical staining of formalin-fixed paraffin-embedded sections using a peroxidase-antiperoxidase (PAP) method demonstrated the presence of glucagon only. Staining reactions for insulin, gastrin, somatostatin and ACTH were negative. The pituitary was microscopically normal.

\section{Discussion}

These two cases eluded accurate diagnosis until postmortem examination was performed. In both instances the observed malakoplakic process was more extensive than the usual case in which the condition is limited to the mucosa of the urinary bladder. Widespread malakoplakia has been described in several cases in which there was clear evidence of hypercorticosteroidism. A case associated with Cushing's syndrome due to ectopic production of ACTH by a bronchial carcinoid is on record..$^{15}$ There have also been reports of cases in which immunosuppression has 


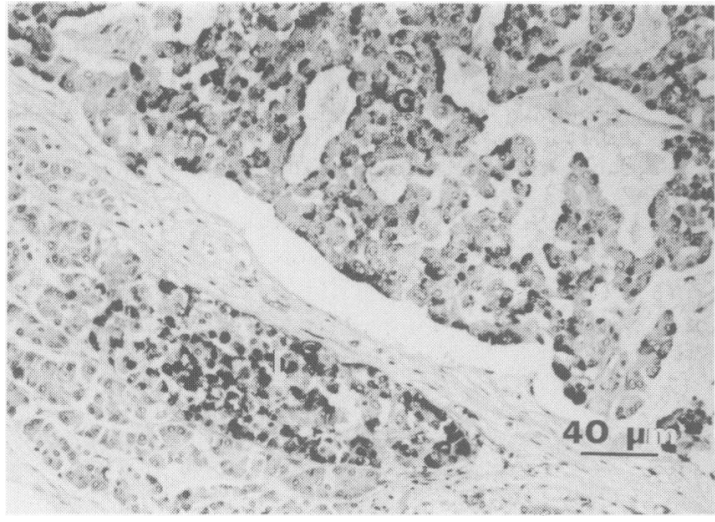

Figure 2 There is a hyperplastic islet of Langerhans (I) in pancreatic tissue adjacent to the glucagonoma (G). The black intracellular material is reaction product indicating the presence of glucagon (PAP).

been produced therapeutically by exogenous corticosteroids. Examples include immunosuppression after cardiac $^{11}$ and renal transplantation ${ }^{6}$ and the use of steroids in the treatment of lymphomas ${ }^{11,14}$ and haemolytic anaemia. ${ }^{12}$

The present two cases demonstrate further evidence of a link between adrenal hyperactivity and malakoplakia. The combined adrenal gland weights (70 and $80 \mathrm{~g}$ ) were approximately six and seven times greater than would be expected in the patients' age group. There was bilateral cortical nodular hyperplasia evident both macro- and microscopically. This affected all the cortex of the four glands studied and raises the possibility that there was stimulation by ACTH although a source of excess ACTH was not detected at necropsy. The evidence that there was adrenal hyperactivity is essentially morphological. A diagnosis

\section{References}

1. Michaelis, L. \& Gutmann, C. Ueber Einschlusse in Blasentumouren. Z Klin 1902, 47: 208-215.

2. McClure, J., Cameron, C.H.S. \& Garrett, R.G. The ultrastructural features of malakoplakia. J Pathol 1981, 134: 13-26.

3. Voight, J. Malacoplakia of the urinary tract. Review of the literature. Report of two new cases. Acta Pathol Microbiol Scand 1958, 44: 377-391.

4. Smith, B.H. Malacoplakia of the urinary tract. Am J Clin Pathol 1965, 43: 409-417.

5. McClure, J. Malakoplakia of the urinary tract. Br J Urol 1982, 54: 181-187.

6. McClure, J. Malakoplakia. J Pathol 1983, 140: 275-330. of Cushing's disease was not made in life.

The co-existence of malakoplakia and a glucagonoma has not been previously documented. It is not possible to decide if this is fortuitous but both are rare, making a chance association very unlikely. It is interesting that there was also a diffuse hyperplasia of pancreatic islet tissue and this might be part of a hyperplastic process involving pancreatic and adrenal endocrine tissue and that the common factor in both cases was the adrenal hyperplasia.

If the malakoplakia is associated with hypercorticosteroidism then it is necessary to consider the mechanisms of this association. It would appear that malakoplakic macrophages are functionally disordered in that their exocytotic activity in extruding phagolysosomes is diminished. Phagocytosis is seemingly unimpaired but the possibility of impairment of intracellular bactericidal activity is not resolved. The evidence of this is conflicting. ${ }^{16,5}$ Nevertheless it would appear that malakoplakic macrophages become overloaded with phagolysosomes and since these are membrane-bound, active transport of calcium ions into the phagolysosome will occur and at a critical concentration hydroxyapatite crystal formation will take place. The phagolysosomes fuse to form the definitive MG body. ${ }^{2}$ It has been postulated that the failure of phagolysosomal exocytosis is an effect of hypercorticosteroidism ${ }^{6}$ although this idea awaits definitive proof.

Despite these doubts about the precise mechanisms there is emerging a clear body of evidence that malakoplakia is associated with adrenocortical hyperactivity, at least when the malakoplakia is more extensive than is usual. There also arises the interesting possibility that more limited examples of malakoplakia are associated with adrenocortical activity of a degree which impairs macrophage function but which does not lead to an overt Cushing's syndrome.

7. McClure, J. Malakoplakia of the testis and its relationship to granulomatous orchitis. J Clin Pathol 1980, 33: 670-678.

8. McClure, J. Malakoplakia of the prostate. A report of two cases and a review of the literature. J Clin Pathol 1979, 32: 629-632.

9. Terner, J.Y. \& Lattes, R. Malakoplakia of colon and retroperitoneum. Am J Clin Pathol 1965, 44: 20-31.

10. Blumbergs, P.C., Hallpike, J.F. \& McClure, J. Cerebral malakoplaia. J Clin Pathol 1981, 34: 875-878.

11. Colby, T.V., Hunt, S, Pelzmann, K. \& Carrington, C.B. Malakoplakia of the lung. A report of two cases. Respiration 1980, 39: 295-299. 
12. Colby, T.V. Malakoplakia. Two unusual cases which presented diagnostic problems. Am J Surg Pathol 1978, 2: 377-382.

13. McClure, J. Malakoplakia of the gastrointestinal tract. Postgrad Med J 1981, 57: 95-103.

14. Lewin, K.J., Fair, W.R., Steigbigel, R.T., Winbert, C.D. \& Droller, M.J. Clinical and laboratory studies into the pathogenesis of malakoplakia. J Clin Pathol 1976, 29: 534-563.
15. McClure, J., Hadden, D.R., Mudd, D.G. \& Parks, T.G. Adrenocortical hyperactivity with disseminated malakoplakia. J Clin Pathol 1977, 30: 206-211.

16. Abdou, N.I., Napombejara, C., Sagawa, A. et al. Malakoplakia. Evidence for monocyte abnormality correctable by cholinergic agonist in vitro and in vivo. $N$ Engl J Med 1977, 297: 1413-1419. 